\title{
COM Model of SAW Gas Sensor Including the Acoustoelectric Effect
}

\author{
Wei LuO ${ }^{1}$, Linglang Y $u^{1}$, Tao Yang ${ }^{1}$, Xiang Xie ${ }^{1}$, Qiuyun Fu ${ }^{1, *}$, Honglang $L^{2} i^{2,}$ \\ ${ }^{1}$ School of Optical and Electronic Information, Engineering Research Center for Functional Ceramics o \\ $f$ the Ministry of Education, Huazhong University of Science and Technology, Wuhan 430074, China, \\ 2 Institute of Acoustics, Chinese Academy of Sciences, 21 North 4th Ring Road, Haidian District, \\ Beijing, 100190, China. \\ fuqy@mail.hust.edu.cn,Ihl@ioa.ac.cn
}

\begin{abstract}
IMCS2018_Headline"):
Extensive research has been carried out on surface acoustic wave (SAW) gas sensor recently. In this paper, a modified coupling of modes (COM) model including acoustoelectric effect for the conductive SAW gas sensors were presented. The whole device performance and variation due to the conductivity variation can be calculated based on this model. Also, a ladder-type behavior of the center frequency variation versus surface conductivity of the film were founded and explained, which corresponds well with our previous results. The center frequency variation and phase catastrophe point variation are two conventional variable observed during the sensing process. Furthermore, we studied the variation of the phase of the $S_{21}$ parameter with the gas concentration, and found this relationship corresponded with the elementary theory. It was concluded that the phase catastrophe point variation are more suitable for being adopted as the variable during gas sensing test.
\end{abstract}

Key words: surface acoustic wave, SAW sensor, coupling of modes model, acoustoelectric effect

\section{Introduction}

The acoustoelectric effect is the theoretical basis for some SAW gas sensors. Many models can calculate the center frequency (velocity), attenuation variation of SAW devices with the conductivity of sensing films. However, the whole sensor performance couldn't be conducted. In this paper, we presented a COM model including acoustoelectric effect for the conductive SAW gas sensors. The whole device performance due to the conductivity variation can be calculated through it and then two different measurement methods were compared. This model can be further used in the optimization process. A conclusion was made that as monotonous and large scale results can be obtained by monitoring the sensor based on the frequency change of the phase catastrophe point [1].

\section{Model}

For the delay line structure device, three $\mathrm{P}$ matrixes were cascaded to calculate device performance. This model can predict the center frequency and attenuation variation with the conductivity of the sensing film for the SAW gas sensors. Even more, it can get the scattering parameters of the whole devices and explain waveform distortion phenomenon of the Sparameter in the test process.

The results correspond very well with the formal simulation results presented by other researchers. Also, they are consistent with the experimental results. The electro-acoustic effect is a complex phenomenon, not only related to the conductivity of the sensitive film, but also with other parameters. The presented model provides another route to simulate the SAW gas sensor performance and offer a tool for future optimization process [2].

\section{Results and Discussion}

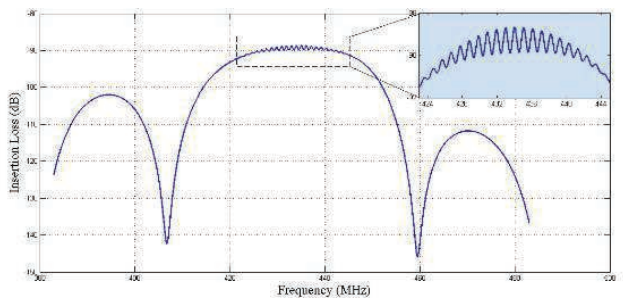

Fig.1. $S_{21}$ curve of SAW device and the phenomenon of passband fluctuation.

Fig. (1) shows the spectral image of insertion loss of the device was obtained by solving the COM mentioned above. 
As it can be seen from the image, the passband fluctuation is obvious. The maximum insertion loss of SAW device with the change of normalized conductivity was calculated as shown in Fig. 2(a). And the change of center frequency with the normalized conductivity was shown in Fig. 2(b). Due to the inherent interfinger and triple reflection effects of the SAW device, the passband fluctuation of the admittance pattern is very conspicuous. During the measurement process, the frequency corresponding to the highest point was usually chose as the center frequency.
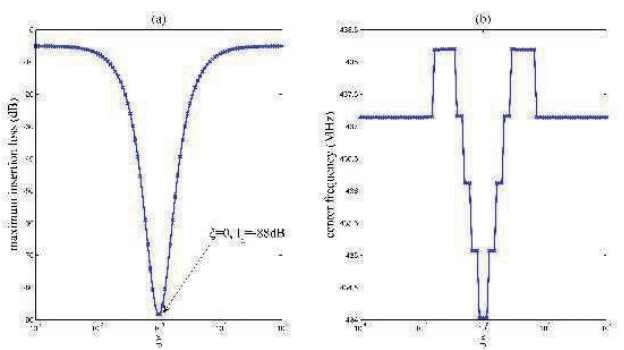

Fig. 2 (a) represents the insertion loss variation with the normalized conductivity. (b) represents the center frequency variation with the normalized conductivity changes.

In fact, passband fluctuation reflects the result of a combination of both the inter-finger reflection effect in the grid region and the triple-reflection transit effect of the propagation region. When the conductivity of the propagation region film changes, the highest peak of the passband fluctuation will change too, accordingly. While the center frequency corresponding to the peak may remain unchanged until the new peak is generated. When the conductivity variation was accumulated to a certain extent, the highest peak of passband fluctuation will be generated from other peaks. Then the center frequency will also shift. That is the reason why a ladder-type behavior of the center frequency of the device is appeared in the Fig. 2(b).

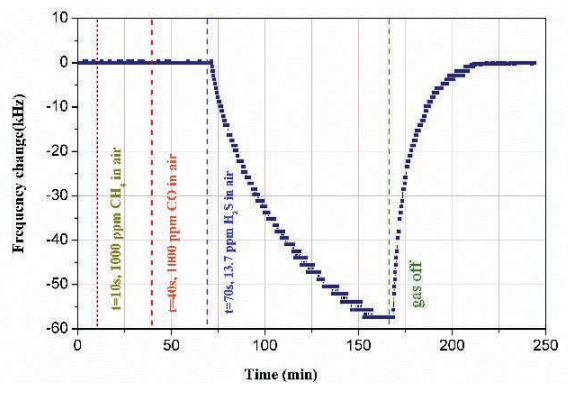

Fig. 3. The similar ladder-type behavior of selective response of SAW sensor to hydrogen sulfide gas [3].

As shown in Fig. 3, the response curve of SAW gas sensor of hydrogen sulfide gas shows similar ladder-type behavior, which shows great consistency with the actual test results to a certain extent [3].
Existence of the non-monotonicity of the center frequencies is very detrimental to actual device testing. Therefore, the trend of the phase of the curve $S_{21}$ was further investigated and found that its variation law was more accurate during the tests than that of the center frequency. Therefore, the method of observing the trend of phase catastrophe point variation was adopted in our study, as shown in Fig. 4.

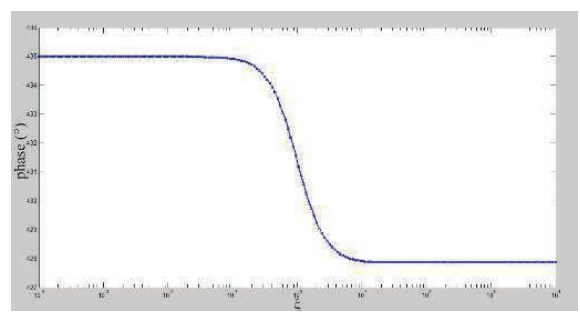

Fig.4. The phase catastrophe point variation of the $S_{21}$ parameters with normalizes the conductivity curve

\section{Conclusion}

In summary, a modified COM model for the conductive SAW gas sensor with acoustoelectric effect being considered has been proposed. Compared to the already existing models, the whole device performance due to the conductivity variation can be calculated based on this model. A ladder-type behavior of the center frequency was observed and explained. It was further found that the phase catastrophe point variation is more suitable for gas sensing test compared to the center frequency variation.

\section{Acknowledgment}

The work was financially supported by the National Natural Science Foundation of China (No. 11574106, 61771448, 51635007), the China Postdoctoral Science Foundation under Grant no. 2017M622404.

\section{References}

[1] Tasaltin C, et al. Acoustoelectric Effect on the Responses of SAW Sensors Coated with Electrospun $\mathrm{ZnO}$ Nanostructured Thin Film, Sensors, 2012, 12(9):12006-15;doi: $10.3390 / \mathrm{s} 120912006$

[2] Wiesław Jakubik, Elemental theory of a SAW gas sensor based on electrical conductivity changes in bi-layer nanostructures, Sensors \& Actuators $B$ Chemical, 2014, 203:511-516; doi: 10.1016/j.snb.2014.07.016

[3] W Luo, et al. Nanocrystalline $\mathrm{SnO}_{2}$, film prepared by the aqueous sol-gel method and its application as sensing films of the resistance and SAW $\mathrm{H}_{2} \mathrm{~S}$ sensor, Sensors \& Actuators $B$ Chemical, 2015, 217(9-10):119-128; doi: 10.1016/j.snb.2014.10.078 